\section{International Journal of Industrial Engineering and Operations Management (IJIEOM)}

Volume 1, No. 1, May 2019

IEOM Society

International

\author{
pp. 1 - 11
}

\title{
Integrating Topological and Hydraulic Attributes for Robustness Analysis of Water Distribution Networks
}

\author{
Seyed Ashkan Zarghami, Indra Gunawan and Frank Schultmann \\ Entrepreneurship, Commercialization \& Innovation Centre (ECIC) \\ The University of Adelaide, Australia \\ ashkan.zarghami@adelaide.edu.au \\ https://doi.org/10.46254/j.ieom.20190101
}

\begin{abstract}
Researchers are recognizing that the robustness evaluation of Water Distribution Networks (WDNs) is of great importance for reducing the impact of disruptive events. Yet, very few methods to measure the robustness of WDNs have been developed. These methods mainly focus on either the topological features or the hydraulic attributes of WDNs and fail to provide a comprehensive picture of the robustness characteristics of WDNs. The work described herein proposes a new robustness index to measure the heterogeneity of WDNs drawing on informational entropy theory. The paper attempts to shift away from an exclusive topological viewpoint or a pure hydraulic approach, towards a combined topological and hydraulic analysis. The main emphasis is on the influence of an individual node on the overall network performance. The use of the proposed index is illustrated with a real-world WDN of an Australian town. The results highlight the significance of integrating the topological and hydraulic metrics for a reliable assessment of robustness in WDNs.
\end{abstract}

ARTICLE INFO

\author{
Received \\ May 1, 2018 \\ Received in revised \\ form \\ May 2018 \\ Accepted \\ June 22, 2018 \\ KEYWORDS \\ Degree distribution; \\ Demand fraction; \\ Informational entropy; \\ Robustness; Water \\ distribution networks.
}

\section{Introduction}

Water Distribution Networks (WDNs) are among the most significant critical infrastructures with a high degree of complexity. Disruption and dysfunction of WDNs would have debilitating effects on different aspects of human life such as safety, economic, security, public health and social well-being (Gunawan et al., 2017). In this respect, it is imperative to have proper measures to evaluate the robustness of WDNs in order to reduce the impact of disruptive events.

There is no unique interpretation of the robustness of complex networks. Extant literature has presented multiple and rather contradicting definitions of robustness. Carlson and Doyle (2002) define the robustness as the ability of a system to fulfill its desired characteristics despite fluctuations in its components behavior. In the work of Li et al. (2008), robustness is found as the ability of the system to avoid malfunction when a fraction of its components are failed. Cuadra et al. (2015) regard the robustness in complex networks as the opposite of vulnerability, which accounts for the reliability of the system. Ferrario et al. (2016) define the robustness as the capability of the network to provide the required level of supply of good when the system is exposed to partial failures. Robustness is also defined as the ability 
of a network to cope with faults during operations (Agathokleous et al., 2017). Although a broad definition of robustness exists, this work addresses the question of system robustness based on Iyer et al. (2013) by analyzing how the network structure and function change as a node is removed.

In recent years, a great deal of research has been conducted on evaluating the robustness of complex networks. Wang et al. (2014) quantified the influence of adding and removing links on the robustness of the complex infrastructural networks by using a graph-based metric. Schieber et al. (2015) proposed a metric for network robustness based on an information theory quantifier by measuring dissimilarities between topologies after each time step of the sequence. Lin et al. (2010) viewed the variability of the degree distribution of the nodes as a key determinant of the network structure. Many researchers have utilized the entropy of degree distribution in order to measure the heterogeneity and therefore robustness of complex networks (Jiang et al. 2014). The entropy of degree distribution as a measure of the network's heterogeneity was computed in the work of Sole and Valverde (2004). Wang et al. (2006) and Wu et al. (2007) investigated the relationship between the entropy of the degree distribution in scale-free networks and the robustness of these networks.

Although researchers have explored innovative methods to quantify the robustness of complex networks, a few methods to measure the robustness of WDNs has been developed hitherto. These methods can be broadly classified into two distinct categories: topological, and hydraulic. In the topological methods, the network robustness is assessed by using a graph invariant from graph theory. Yazdani and Jeffrey (2012a, 2012b) are among a few researchers that quantified the robustness of WDNs by using graph invariants such as meshedness coefficient, spectral gap, and algebraic connectivity metrics. Most existing literature has studied the robustness of WDNs along with the network reliability by taking a hydraulic viewpoint. For example, Greco et al. (2012) developed an entropy-based demanddriven approach for measuring the robustness of WDNs. Jung et al. (2014) proposed a robustness index by measuring the variation of the system hydraulic performances. Agudelo-Vera et al. (2014) examined the robustness of drinking WDNs based on two main hydraulic parameters: minimal pressure and water quality presented as water age.

The research just cited is embedded in embracing either the topological features or the functional attributes of WDNs and therefore have particular strengths and weaknesses. On the one hand, pure topological measurements of the robustness WDNs are useful to describe the network structure, but they fail to properly characterize the network properties (Yazdani and Jeffrey, 2011). On the other hand, relying solely on the hydraulic properties of WDNs hinders a modeler to evaluate the robustness inherent within the layout of the network.

The overarching aim of this research is to shift away from a pure topological perspective or an exclusive hydraulic viewpoint towards a combined topological and hydraulic analysis in order to present a more accurate measure of the WDNs robustness. We advocate the idea that an extensive evaluation of the network robustness depends not only on the topological measurements but also on the functional attributes of the network (Yazdani et al., 2011). It is in this spirit that this work proposes a combined topological and hydraulic metric for measuring the robustness of WDNs based on the principle that a network with uniformly distributed values of the required demand along with an equiprobable distribution of the nodal degree sequence, exhibits a lower drop in performance in the case of partial failure of nodes. To this end, the paper develops a methodology, using informational entropy theory, for evaluating the robustness of WDNs. The research focuses on a joint entropy model by coupling two entropy metrics: degree distribution entropy-based metric denoting the topological attributes of the nodes and demand fraction entropy-based metric representing the hydraulic attributes of the nodes in the network.

The proposed robustness analysis method differs from the conventional methods along three key dimensions. First, in contrast to the conventional methods in which the robustness of a WDN is mainly assessed based on the network reliability, this research addresses the robustness of a network by removing a node and subsequently measuring its influence on the overall performance of the network. This is precisely consistent with the preceding definition of the robustness. Second, by situating our research in the informational entropy context, we demonstrate how evaluating the statistical diversity of the hydraulic and topological attributes of WDNs can be interpreted as a measure of the network robustness. Third, this research gives a vivid account of the network robustness by taking into account the joint consideration of the nodal demands, as a hydraulic attribute, along with the nodal degree distribution, as a structural property.

In what follows, Section 2 reviews the degree distribution and demand fraction entropy-based metrics, followed by developing a joint entropy-based index to measure the robustness of WDNs. In Section 3, the proposed robustness index is applied to a real-world case study. Section 4 is devoted to a discussion of the results obtained from the proposed method. The paper is concluded in Section 5 and areas for further research are discussed in the end. 


\section{Provenance of Entropy-Based Robustness Index}

To begin to assess the robustness of WDNs, first, the notion of informational entropy is briefly described, which can be leveraged to evaluate the robustness of the network.

The classic definition of informational entropy attributed to Shannon (1948) measures choice and uncertainty. Shannon entropy of a set of probabilities $P=\left\{p_{i}: i=1,2, \ldots, n\right\}$, can be formulated as follow:

$$
H=-\sum_{i=1}^{n} p_{i} \log _{b} p_{i}
$$

where $H$ is entropy of distribution, $n$ is the number of possible outcomes and $b$ is an arbitrary logarithm base by which the unit of entropy is defined. For $b=2, b=e$, and $b=10$, the units are defined as bits, Napier, and decibels, respectively.

The informational entropy has been found to have useful applications in a wide range of areas, including evaluating the reliability and redundancy of WDNs (e.g. Awumah et al., 1991, Tanyimboh, 2017, Zarghami et al., 2018a), hydrology (e.g. Li et al., 2012), transportation networks (e.g. Wu et al., 2013), and medical science (e.g. Sato et al., 2013). In this section, the principle of informational entropy is adopted as the measure of statistical diversity of degree and demand distribution in WDNs. The remainder of this section demonstrates how the diversity of variables, obtained as results of informational entropy, can be interpreted in terms of the network robustness.

\section{Entropy of Degree Distribution}

We now adopt a graph theory quantity known as the degree of a node. The degree of a node is the number of links that connect the node to other nodes. The degree distribution, $g_{k}$, is the probability that a randomly chosen node has $k$ links. Valuable information on intrinsic network structure can be inferred from the node degree distribution (Pozrikidis, 2016). As discussed earlier, the entropy of degree distribution has been widely used as a measurement of robustness for complex systems. Here this notion is recalled so as to provide helpful clarification.

Let $k_{i}$ be the degree value of node $i$ and let $\left\{g_{1}, g_{2}, \ldots, g_{\Delta}\right\}$ define the degree sequence distribution of the network, where $g_{k}$ is the fraction of nodes with degree $k$. The entropy of degree distribution of a network, that is, $H_{d}$, is a measure of heterogeneity in the network, which can be formulated as:

$$
H_{d}=-\sum_{k=1}^{\Delta} g_{k} \log _{2}\left(g_{k}\right)
$$

where $\Delta$ represents the maximum degree of nodes in the network. The higher value of $H_{d}$ denotes more diversity in link distribution. Intuitively, diversity in degree distribution increases the robustness of the network, thus the higher the entropy of the degree distribution, the more robust of the network is. $H_{d}$ attains its maximum value $\left(\log _{2} \Delta\right)$ when $g_{k}=$ $\frac{1}{\Delta}$ for any node degree $k$, and its minimum value 0 when the node degrees are either 0 or 1 .

Despite a broad use of the degree distribution entropy-based metric in assessing the robustness of networks, $H_{d}$ only captures very generic topological information of the complex networks and therefore is poorly informative. A reliable robustness assessment of WDNs requires further specification of topological and hydraulic features of the network. Moreover, in WDNs the maximum nodal degree is generally very low because such networks are located on a twodimensional space, which are constrained, by a number of impediments, therefore the results obtained from the standalone use of the entropy-based degree distribution metrics might be unreliable (Giustolisi et al., 2016).

\section{Entropy of Demand Fraction}

With the aim of addressing the aforementioned shortcoming of the degree distribution entropy-based metric, the robustness evaluation of WDNs is now extended by proposing a new hydraulic measurement, called demand fraction entropy-based robustness metric, $\left(H_{q}\right)$.

Let a network consists of $N$ nodes and let the required flow (demand) at node $i$ be denoted by $q_{i}$. For node $i$, the parameter $p_{i}$ in Eq. (1) is defined as:

$$
p_{i}=\frac{q_{i}}{Q_{t}}
$$


where $p_{i}$ is demand fraction at node $i$, and $Q_{t}$ is the total required flow of the network and given by:

$$
Q_{t}=\sum_{i=1}^{N} q_{i}
$$

The parameter $p_{i}$ can be interpreted as a probability value for $q_{i}$. It represents the contribution of node $i$ to the total flow of the network. To develop the demand fraction entropy-based robustness metric, Eq. (1) can be restated as follows:

$$
H_{q}=-\sum_{i=1}^{N}\left(\frac{q_{i}}{Q_{t}}\right) \log _{2}\left(\frac{q_{i}}{Q_{t}}\right)
$$

Since $\sum_{i=1}^{N} p_{i}=1$, the variables of the set $P=\left\{p_{i}: i=1,2, \ldots, n\right\}$ are conditionally reliant on each other. That is, if the demand fraction at a particular node increases, the summation of all other variables tends to decrease and vice versa. From the informational entropy perspective, thus, $H_{q}$ can be constructed as a measure of the network robustness that indicates the degree of severity of the single failure of nodes. The intuitive interpretation of $H_{q}$ is that a critical node with the highest value of demand fractions contributes more to the drop in overall network performance in response to random failures. In fact, removal of a node with a high demand fraction leaves a larger number of households without water supply in comparison to the failure of nodes with lower demand fractions. Thus, it is more advantageous from the robustness point of view, if all nodes are of equal value of demand fraction.

\section{Joint Entropy of Degree Distribution and Demand Fraction}

To achieve a reliable assessment of the robustness, the authors argue that the focus should shift away from an exclusive topological viewpoint or a pure hydraulic approach, towards a combined topological and hydraulic analysis. To this end, the paper proposes a new index drawing on the joint entropy of degree distribution and demand fraction in order to measure the robustness of WDNs.

The joint entropy, $H\left(X_{1}, X_{2}\right)$, of a pair of random variables with a set of joint probability of $\left\{p_{i j}: i=1,2, \ldots, N ; j=1,2, \ldots, M\right\}$, is defined as:

$$
H\left(X_{1}, X_{2}\right)=-\sum_{i=1}^{N} \sum_{j=1}^{M} p_{i j} \log _{2}\left(p_{i j}\right) \leq H\left(X_{1}\right)+H\left(X_{2}\right)
$$

Demand fraction and degree distribution are stochastically independent variables, hence their joint entropy can be obtained from the following equation (Singh, 2013):

$$
H(d, q)=H_{d}+H_{q}
$$

For a network with $N$ nodes and the maximum degree of $\Delta, H(d, q)$ is maximum when $g_{i}=\frac{1}{\Delta}$ and $q_{i}=\frac{Q_{i}}{N}$, hence:

$$
H_{\text {max }}(d, q)=-\left(\log _{2} \Delta+\log _{2} N\right)
$$

The robustness index, $R S_{I}$, is defined as the fractional differences between $H_{\max }(d, q)$ and $H(d, q)$, which can be expressed by the following equation:

$$
R S_{I}=\frac{H(d, q)}{H_{\max }(d, q)}
$$

Our robustness index combines both the degree distribution and the demand fraction and can be obtained by substituting Eq. (2), Eq. (5) and Eq. (8) into Eq. (9) as:

$$
R S_{I}=\frac{\sum_{k=1}^{\Delta} g_{i} \log _{2}\left(g_{i}\right)+\sum_{i=1}^{N}\left(\frac{q_{i}}{Q_{t}}\right) \log _{2}\left(\frac{q_{i}}{Q_{t}}\right)}{\log _{2} \Delta+\log _{2} N}
$$

The robustness index proposed here is the ratio of the network robustness to the maximum possible robustness, which describes the distance to the degree of severity of failures in the network, due to the failure of each individual node. $R S_{I}$ falls within the range of $[0,1]$ and satisfies the following properties:

1) $R S_{I}$ is a dimensionless value, which implies the relative entropy. 
2) When $R S_{I}$ is closer to 1 the robustness is higher, and otherwise is smaller.

3) $R S_{I}$ attains its maximum value $\left(R S_{I}=1\right)$ when $\left\{g_{k}=\frac{1}{\Delta}: k=1,2, \ldots, \Delta\right\}$ and $\left\{p_{i}=\frac{Q_{t}}{N}: i=1,2, \ldots, N\right\}$.

\section{A Summary of the Research Design}

This research maps a WDN into a graph of nodes and edges. The edges of the graph represent the pipes of a WDN and nodes represent the demand nodes through which water is supplied to the consumers. Each node in the graph is associated with its degree as well as its required flow. The contribution of a node $i$ to the robustness of the network is first estimated by calculating the degree of the node $(k)$ along with the relative importance of the node to the total required water for the network $\left(p_{i}\right)$. The diversity in degree distribution as a proxy to estimate the topological diversity of the network is then measured by calculating the entropy of the probability distribution of the fraction of nodes with various degrees $\left(g_{k}\right)$ by using Eq. (2). In a similar vein, the entropy of the set of demand fraction values is calculated by using Eq. (5). We then proceed with evaluating the joint consideration of the topological and hydraulic robustness of the network. The robustness of the network as a whole is obtained from the joint entropy of a combination of the entropy degree distribution and demand fraction by using Eq. (7). Finally, the robustness index $\left(R S_{I}\right)$ is constructed based on the fractional differences between the joint entropy and the maximum achievable entropy using Eq. (10). The elaborated structure of the proposed method is demonstrated on a real-world case study in the following section.

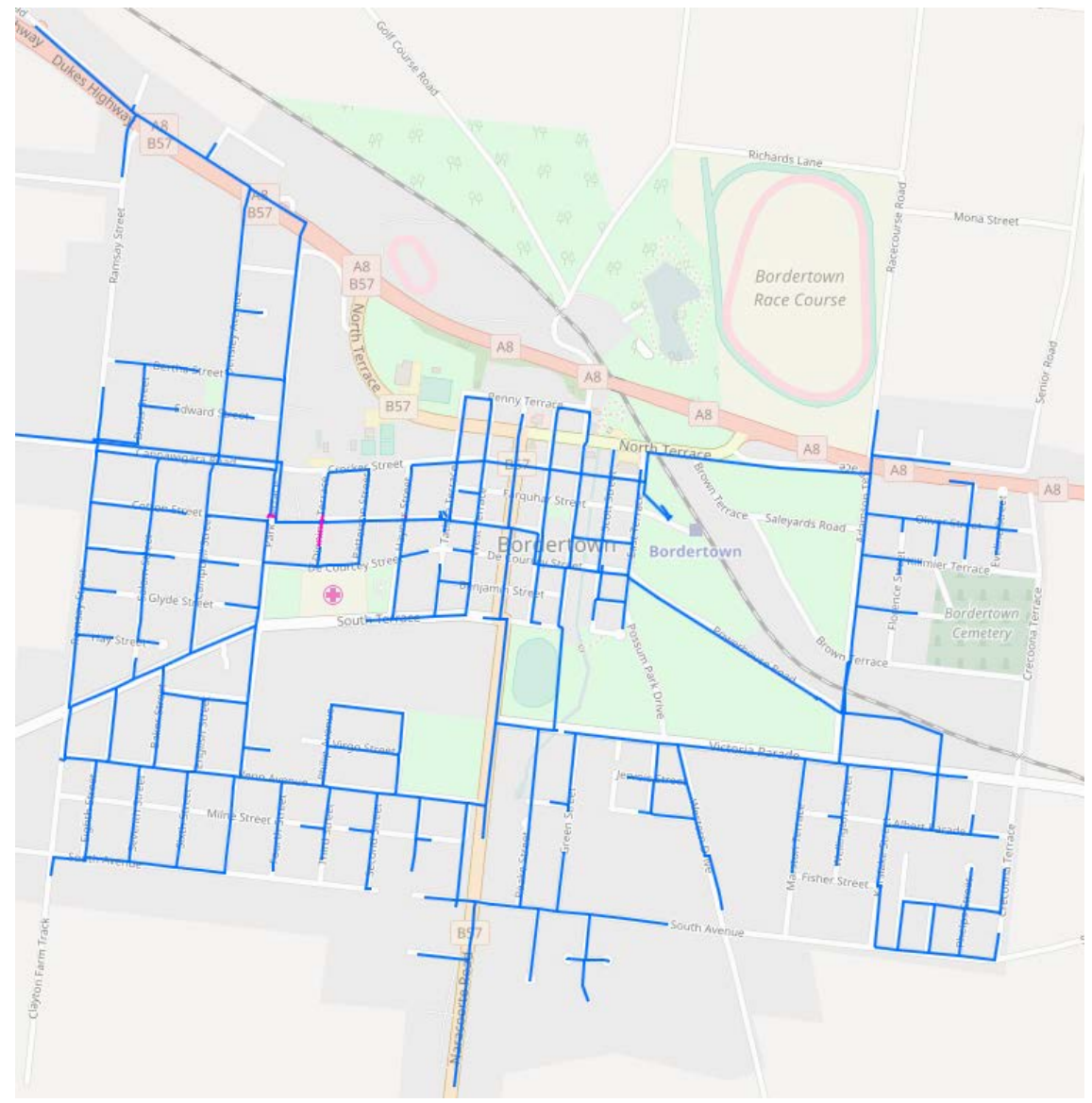

Figure 1. Bordertown water distribution network

\section{Application}

In order to compute the value of the robustness index, discussed in section 2, it is now time to turn to a real-world WDN of Bordertown, an Australian town supplying 2800 inhabitants in South Australia near the Victorian border (Fig. 1). The data for this research has been obtained from the official website of SA Water, containing the layout of the network, 
pipes diameter, and the number of households connected to each pipe (http://sawater.maps.arcgis.com). The example network is mapped into a graph with 216 nodes, representing demand nodes, and 256 edges, representing pipes varying from $80 \mathrm{~mm}$ to $375 \mathrm{~mm}$.

Results from the application of the proposed method to data from the case study are now presented. We start with computing the entropy of degree distribution $\left(H_{d}\right)$ and then proceed with obtaining the demand fraction entropy $\left(H_{q}\right)$. The robustness index $\left(R S_{I}\right)$ is finally developed using the values of $H_{d}, H_{q}$, and the maximal entropy.

The parameters used for computing $H_{d}$ are listed in Table. 1.

Table 1. The parameters used for obtaining $H_{d}$

\begin{tabular}{ccccc}
\hline$k$ & $n_{k}$ & $g_{k}=\frac{n_{k}}{N}$ & $\log \left(g_{k}\right)$ & $g_{k} \log \left(g_{k}\right)$ \\
\hline 1 & 74 & 0.3426 & -1.5454 & -0.5295 \\
2 & 25 & 0.1157 & -3.1110 & -0.3601 \\
3 & 84 & 0.3889 & -1.3626 & -0.5299 \\
4 & 33 & 0.1528 & -2.7105 & -.04141 \\
\hline
\end{tabular}

By substituting the results from Table 1 into Eq. (2), $H_{d}=1.8335$ bits.

A similar procedure can be used for calculation of $H_{q}$. Due to a large number of nodes, Table 2 shows a part of the parameters used for calculating $H_{q}$ representatively.

Table 2. Some of the parameters used for obtaining $H_{q}$

\begin{tabular}{cccc}
\hline Node & $p_{i}=\frac{q_{i}}{Q_{t}}$ & $\log \left(\frac{q_{i}}{Q_{t}}\right)$ & $\frac{q_{i}}{Q_{t}} \log \left(\frac{q_{i}}{Q_{t}}\right)$ \\
\hline 1 & 0.00154 & -9.3469 & -0.01435 \\
2 & 0.00154 & -9.3469 & -0.01435 \\
3 & 0.00307 & -8.3469 & -0.02564 \\
4 & 0.00324 & -8.2689 & -0.02681 \\
$:$ & $:$ & $:$ & $:$ \\
105 & 0.00234 & -8.7392 & -0.02045 \\
106 & 0.00586 & -7.4153 & -0.04344 \\
$:$ & $:$ & $:$ & $:$ \\
215 & 0.00341 & -8.1949 & -0.02797 \\
216 & 0.00171 & -9.1949 & -0.01569 \\
\hline
\end{tabular}

By using Eq. (5), $H_{q}=7.5604$ bits.

Given $\Delta=4$ and $N=216$, now is possible to calculate the robustness index of the example network, using Eq. (10), that is, $R S_{I}=0.9630$.

$R S_{I}$ can be interpreted as a measure of distance from the maximum possible entropy of the network. The high value of $R S_{I}$ in the example network implies low heterogeneity and subsequently high level of serviceability in the case of random failure of a node.

For completeness, the results of this analysis are summarized in Table 3. 
International Journal of Industrial Engineering and Operations Management (IJIEOM)

Volume. 1, No. 1, pp. 1-11, May 2019

Table 3. The number of nodes, maximum nodal degree, the entropy of degree distribution, the entropy of demand fraction, robustness index

\begin{tabular}{cccccc}
\hline$N$ & $\Delta$ & $H_{d}$ & $H_{q}$ & $H_{\max }(d, q)$ & $R S_{I}$ \\
\hline 216 & 4 & 1.8335 & 7.5604 & 9.7546 & 0.9630 \\
\hline
\end{tabular}

\section{Discussion}

We now compare a few key characteristics of nodal degree distribution and demand fraction concepts. Visualizations underlying the value of $R S_{I}$ are also presented here.

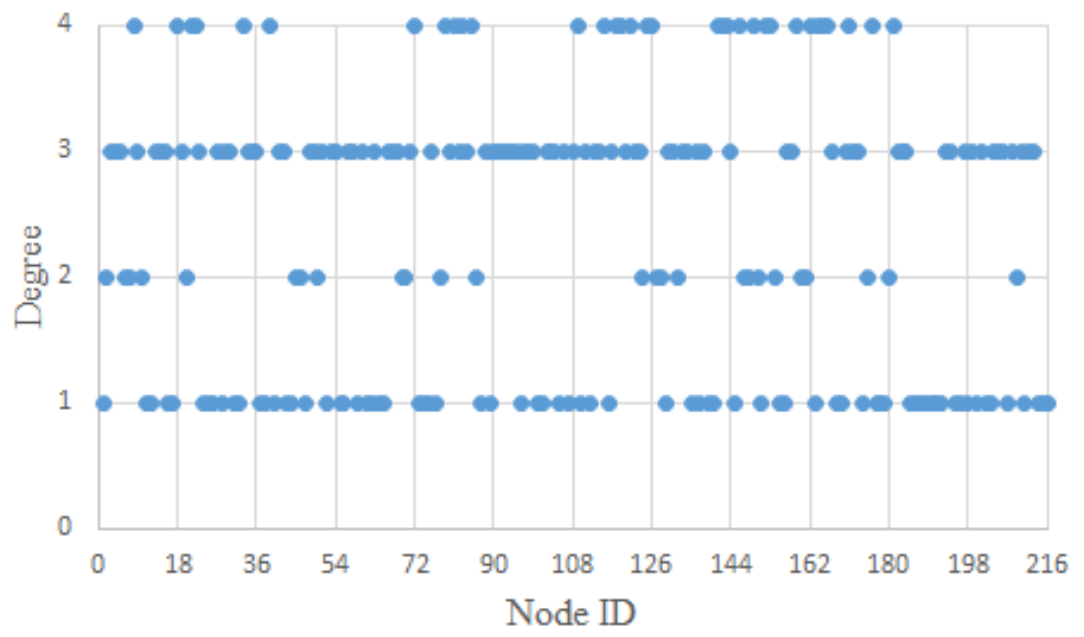

(a)

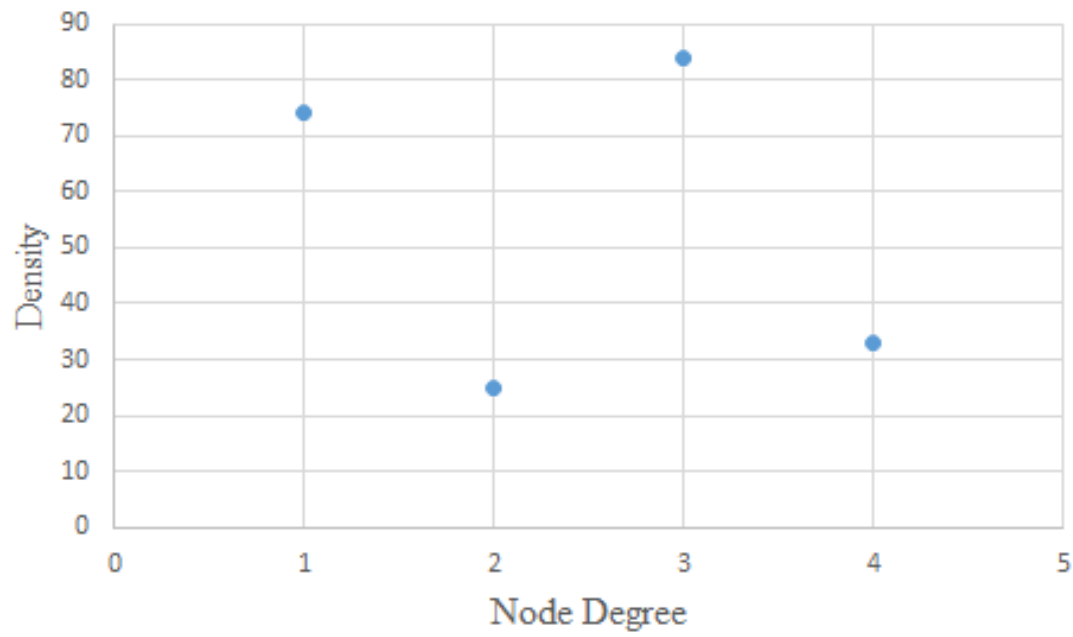

(b)

Figure 2. (a) Degree of each node; (b) Nodal degree distribution of the example network

Figure 2(a) shows the degree of nodes in the example network. A low diversity of the node degrees is observed such that the maximum degree is four. Consequently, only four points can be considered for obtaining the degree distribution entropy. Fig. 2(b) depicts the density against the nodal degree. The nodes have a maximum of 4 links and a minimum of one link. Degree 3 and 1 nodes are the most occurring degrees in the network, whereas degree 2 and 4 are the less frequent degrees. As expected for a real-world WDN, a low heterogeneity of the nodal degree values was observed. This result is consistent with the two well-known facts. First, for WDNs, the nodal degree distribution spans over a very 
limited range (in this case from, 1 to 4). Second, the maximum nodal degree for WDNs is generally very low (Giustolisi et al., 2016) and therefore yields insufficient information for measuring the robustness of a WDN.

By contrast, as reported in Figure 3(a), the demand fractions span a wider range of values, ranging from 0.00068 to 0.01501 . As discussed in the preceding section, incorporating more variables into the probabilistic model entails a more reliable estimation of the robustness. Moreover, as shown in Figure 3(b), it is observed that the density values are more frequent around the corresponding value of the uniformed demand distribution, that is, $\frac{1}{N}=0.0046$, which implies homogeneity of the network from the demand distribution perspective.
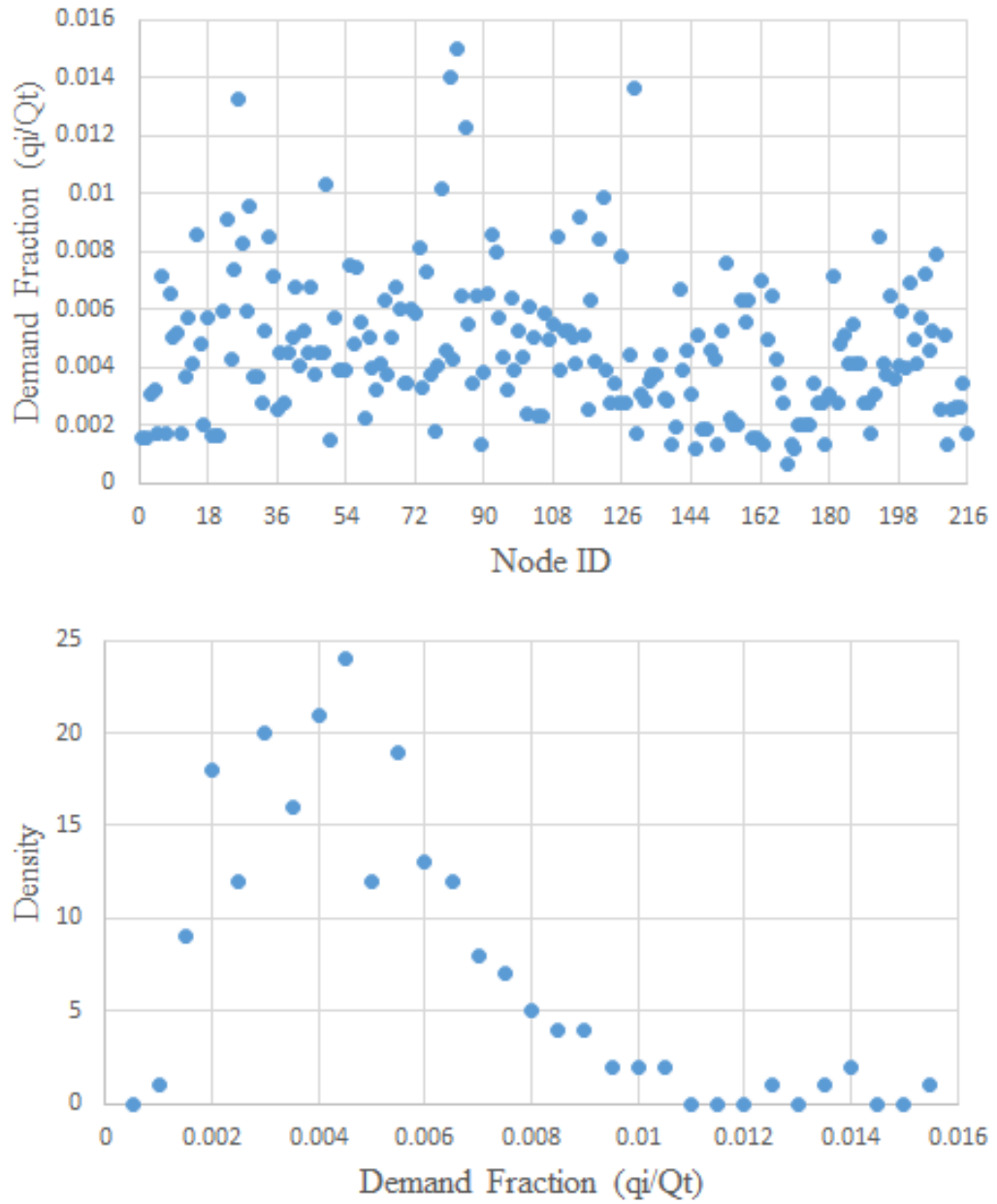

(b)

Figure 3. (a) Demand fraction of each node; (b) Demand fraction distribution of the case study

Figure 3(a) plots the scatterplot of $p_{i}$, illustrating the level of heterogeneity in the network. As Giustolisi et al., (2008) put forward, the fraction of unsupplied demand is a good indicator of the overall network performance. Thus, the demand fraction can be interpreted as a measure for the loss of the network performance when a node is failed. As shown in Figure 3(a), the loss of performance oscillates between $0.07 \%$ and $1.5 \%$. In terms of the demand fraction values, the nodes can be broadly grouped as follows. The nodes with $p_{i}>0.01$, whose failures lead to more than $1 \%$ loss of the network performance and the nodes with $p_{i}<0.01$, whose failures result in less than $1 \%$ loss in the network performance. The single failure of 209 out of 216 nodes in the network (the latter group), results in loss of overall performance at values of less than $1 \%$, thereby confirming low heterogeneity of the studied network. More precisely, the removal of nodes $26,49,79,81,83,85$, and 129 with relatively large values of $p_{i}$ will have more consequences on the network performance. In the other words, failure of these seven nodes accounts for $8.87 \%$ loss of the network performance. 
As above discussion attests, the high value of $R S_{I}=0.963$ is not entirely surprising and, indeed this value supports the fact that the Bordertown WDN is a spatially organized network with low heterogeneity, where most of the nodes have comparable influence form the degree and the demand perspectives. In fact, $R S_{I}$ measures the degree of operability of the network due to the failure of each individual nodes. The larger $R S_{I}$ the lesser the consequences of the nodal failure on the network performance.

\section{Conclusion}

The method described herein represents a new step toward measuring the robustness of WDNs. The authors advocate the idea that the behavior of a network can be characterized by assessing the influence of each individual node in the network (Singh et al., 2015). Different authors have developed either topological perspectives or hydraulic methods to evaluate the robustness of WDNs. The present research emphasizes that persistent focus on topological properties or exclusive emphasize on hydraulic attributes of WDNs fails to realistically measure the robustness. To remedy this weakness, rooted in entropy theory, a new robustness index is proposed, which combines the topological and hydraulic attributes of the network. The proposed index not only explores the structural heterogeneity of the network by using an entropy-based degree distribution metric, but also incorporates the demand fraction entropy that accounts for the hydraulic attribute of WDNs. This integrated approach yields more useful insights to evaluate the robustness of WDNs in comparison with the conventional approaches.

The paper posits the informational entropy theory as a tool to measure the robustness of WDNs. The work also measures and analyses the robustness of a network from the character of its heterogeneity (Wang et al., 2006). On this premise, the joint entropy of degree distribution and demand fraction is employed to measure the network's heterogeneity, which in turn can be interpreted as the measure of robustness. The entropy-based analysis reveals that a uniform demand distributed network with diversity in its degree distribution is less heterogeneous and thus exhibits more robust behavior against the random failure of its nodes.

The use of the proposed index was illustrated with a real-world case study. The paper compared the effectiveness of the demand fraction entropy-based metric with the degree distribution entropy-based metric. The numerical results confirmed that the nodal degrees in WDNs span over a small range of values, consequently the degree distribution entropy-based metric is not informative enough and therefore the stand-alone use of this metric hinders a reliable assessment of the robustness in WDNs (Giustolis et al., 2017).

Two practical implications can be drawn from the robustness analysis method proposed in this research. First, the model developed in this work helps the water utilities to design an effective risk mitigation plan by eliciting the knowledge about how the network continues to perform after failures of its individual nodes. Second, the model can be used as a complement to the conventional reliability based maintenance models by means of measuring the degree of consequences of the nodal failures in WDNs.

The current study is not exempt from limitations. Water demand is a non-stationary attribute that changes over time. The proposed robustness index provides a snapshot of the network at a particular point in time. Therefore, as suggested by Zarghami et al., (2018b), there is a need for a complementary approach (e.g., system dynamics) to design a model that can be updated as the nodal water demands change over time. Furthermore, though the degree distribution and demand fraction are good indicators of the topological and the hydraulic (respectively) performance of WDNs, these metrics do not entirely characterize the structural and hydraulic behaviors of the network. Further research can build upon our current study by incorporating more topological (e.g. centrality measures) as well as hydraulic (e.g. pressure head at nodes) metrics into our method. We believe that the robustness evaluation of WDNs requires incorporating more factors than is presented in this paper and hope the current study further encourages researchers to develop more innovative methods.

\section{References}

Agathokleous, A., Christodoulou, C., and Christodoulou, SE., Topological robustness and vulnerability assessment of water distribution networks, Water Resource Management, pp. 1-15, 2017.

Agudelo-Vera, C., Blokker, M., Vreeburg, J., Bongard, T., Hillegers, S., and Van Der Hoak, JP., Robustness of drinking water distribution networks under changing failure demand, Procedia Engineering, vol. 89, pp. 339-346, 2014. 
International Journal of Industrial Engineering and Operations Management (IJIEOM)

Volume. 1, No. 1, pp. 1-11, May 2019

Awumah, K., Goulter, I., and Bhatt, SK., Entropy-based redundancy measures in water distribution networks. Journal of Hydraulic Engineering. vol. 117, no. 5, pp. 595-614, 1991.

Carlson, JM., and Doyle, J, Complexity and robustness Proceedings of the National Academy of Sciences of the United States of America, vol. 99, pp. 2538-2545, 2006.

Cuadra, L., Salcedo-Sanz, S., and Del Ser, J., Jimenez-Fernandez, S., Geem, ZM., A critical review of robustness in power grids using complex networks concept, Energies, vol. 8, no. 9, pp. 9211-9265, 2015.

Ferrario, E., Pedroni, N., and Zio, E., Evaluation of the robustness of critical infrastructures by hierarchical graph representation, clustering and Monte Carlo simulation, Reliability Engineering and System Safety, vol. 155, pp. 78-96, 2016.

Giustolisi, O., Kapelan, Z., and Savic, D., Extended period simulation analysis considering valve shutdowns, Journal of Water Resources Planning and Management, vol. 134, no. 6, pp. 527-537, 2008.

Giustolisi, O., Simone, A., and Ridolfi, L., Classification of infrastructure networks by neighbourhood degree distribution, arXiv preprint arXiv: 1609.07580, 2016.

Giustolisi, O., Simone, A., and Ridolfi, L., Network classification and features of water distribution systems, Water Resources Research, vol. 53, no. 4, pp. 3407-3423, 2017.

Greco, R., Di Nardo, A., and Santonastaso, G., Resilience and entropy as indices of robustness of water distribution networks. Journal of Hydroinformatics, vol. 14, no. 3, pp. 761-771, 2012.

Gunawan, I., Schultmann, F., and Zarghami, SA., The four Rs performance indicators of water distribution networks: A review of research literature, International Journal of Quality \& Reliability Management, vol. 34, no. 5, pp. 720-732, 2017.

Iyer, S., Killingback, T., Sundaram, B., and Wang, Z., Attack robustness and centrality of complex networks, PLoS ONE, vol. 8, no. 4, e59613, 2013.

Jiang, Y., Hu, AQ., and Song, YB, The evaluation of complex networks' robustness based on entropy measure. In Communications Security Conference, pp. 1-5, 2014.

Jung, D., Kang, D., Kim, JH., and Lansey, K., Robustness-based design of water distribution networks, Journal of Water Resources Planning and Management, vol. 140, no. 11, pp. 04014033:1-14, 2014.

Li, C., Singh, VP., and Mishra, AK., Entropy theory-based criterion for hydrometric network evaluation and design: Maximum information minimum redundancy, Water Resource Research, vol. 48, no. 5, W05521:1-15, 2012.

Li, P., Wang, BH., Sun, H., Gao, P., and Zhou, T., A limited resource model of fault-tolerant capability against cascading failure of complex networks, The European Physical Journal B, vol. 62, no. 1, pp. 101-104, 2008.

Lin, Y., Desouza, KC., and Roy, S., Measuring agility of networked organizational structure via network entropy and mutual information, Applied Mathematics and Computation. vol. 216, no.10, pp. 2824-2836, 2010.

Pozrikidis, C., Node degree distribution in spanning trees, Journal of Physics A: Mathematical and Theoretical. vol. 49, no.12, p. 125101, 2016.

SA Water, Water Mains Replacement Program, viewed $15^{\text {th }}$ August 2017, http://sawater.maps.arcgis.com/apps/View/index.html?appid=afdd0293e7834256beb889b205f8b830,

Sato, JR., Takahashi, DY., Hoexter, MQ., Massirer, KB., and Fugita, A., Measuring network’s entropy in ADHD: a new approach to investigate neuropsychiatric disorders, NeuroImage, vol. 77, pp. 44-51, 2013.

Schieber, TA., Carpi, L., Frery, AZ., Rosso, OA., Pardalos, PM., and Ravetti, MG., Information theory perspective on network robustness, Physics Letters A, vol. 380, no. 3, pp. 359-364, 2016.

Singh, P., Chakraborty, A., and Manoj, BS., Link influence entropy, Physica A: Statistical Mechanics and its Applications, vol. 465, pp. 701-713, 2017.

Singh, VP., Entropy Theory and its Application in Environmental and Water Engineering. Wiley-Blackwell, New York, USA, 2013.

Shannon, CE., A mathematical theory of communication, Bell System Technical Journal, vol.27, no.3, pp. 379-423, 1948.

Sole, RV., and Valverde, S., Information theory of complex networks: On evolution and architectural constraints, In Complex Networks, pp. 189-207, Springer, Berlin, Heidelberg, 2004.

Tanyimboh, TT., Informational entropy: A failure tolerance and reliability surrogate for water distribution networks. Water Resources Management. , vol. 31, no. 10, pp. 3189-3204, 2017.

Wang, B., Tang, H., Guo, C., Hio, C., and Zhou, Entropy optimization of the scale-free networks' robustness to random failure, Physica A: Statistical Mechanics and its Applications, vol. 36, no. 2, pp. 591-296, 2006.

Wang, X., Pournaras, E., Kooij, RE., and Van Mieghem P., Improving robustness of complex networks via the effective graph resistance, The European Physical Journal B, vol. 87, no. 9, pp. 1-12, 2014.

Wu, J., Tan, YJ., Deng, HZ., and Zhu, DZ., Heterogeneity of scale-free networks, Systems Engineering-Theory\& Practice, vol. 27, no. 5, pp. 101-105, 2007. 
Wu, L., Tan, Q., and Zhang, Y., Network connectivity entropy and its application on network connectivity reliability, Physica A: Statistical Mechanics and its Applications, vol. 392, no. 21, pp. 5536-5541, 2013.

Yazdani, A., and Jeffrey, P., Complex network analysis of water distribution systems, Chaos: An Interdisciplinary Journal of Nonlinear Science, vol. 21, no. 1, p. 016111, 2011.

Yazdani, A., and Jeffrey, P., Applying network theory to quantify the redundancy and structural robustness of water distribution systems, Journal of Water Resource Planning and Management, vol. 138, no. 2, pp. 153-161, 2012 a.

Yazdani, A., and Jeffrey, P., Water distribution system vulnerability analysis using weighted and directed network models, Water Resource Research, vol. 48, no. 6, 2012 b.

Yazdani, A., Otoo RA., and Jeffrey, P., Resilience enhancing expansion strategies for water distribution systems: A network theory approach, Environmental Modelling \& Software, vol. 26, no. 12, pp. 1574-1582, 2011.

Zarghami, SA., Gunawan I., and Schultmann, F., Integrating entropy theory and cospanning tree technique for redundancy analysis of water distribution networks, Reliability Engineering and System Safety, vol. 176, pp. 102112, 2018 a.

Zarghami, SA., Gunawan I., and Schultmann, F., System dynamics modelling process in water sector: a review of research literature, System Research and Behavioral Science, In press.

\section{Biographies}

Seyed Ashkan Zarghami is a PhD candidate in Faculty of Professions at the University of Adelaide, Australia. He is currently researching on complex systems. He holds a Bachelor's degree in civil engineering, Master's degrees in project management and civil \& structural engineering. Prior to his doctoral studies, he worked for several years in water sector and infrastructure projects.

Indra Gunawan is Associate Professor in Complex Project Management and Director of Project Management Program in the Entrepreneurship, Commercialisation and Innovation Centre at the University of Adelaide, Australia. He received his $\mathrm{PhD}$ in Industrial Engineering and MSc in Construction Management from Northeastern University, USA. His current research interests include system reliability modelling, maintenance optimisation, project management, applications of operations research, and operations management. His work has appeared in many peer-reviewed journals and conference proceedings.

Frank Schultmann holds a Professorship of Business Administration at the Karlsruhe Institute of Technology (KIT) and is director of the KIT's Institute for Industrial Production (IIP) and the KIT's French-German Institute for Environmental Research (DFIU). He is also Professor of Complex Project Management for the University of Adelaide. He completed his doctoral thesis in 1998 and his Habilitation in 2003 at the Faculty of Economics and Business Engineering at the University of Karlsruhe (now KIT). Prior to his present positions he was Professor of Industrial Management at the University of Koblenz-Landau and holder of the Chair of Business Administration, Construction Management and Economics at the University of Siegen. His research interests include sustainable production and logistics, decision support, supply chain management and optimization, systems engineering, project management, technology assessment, construction management, and information and communication technologies. 